\title{
LONG-TERM VARIATIONS OF EARTH'S ORBITAL GEOMETRY AND IT S EFFECT ON THE CLIMATE SYSTEM
}

\author{
YINGMIN HUA \\ Shanghai Observatory, Chinese Academy of Sciences \\ Shanghai 200030 \\ China
}

\begin{abstract}
Chinese Loess data over the past 2.4 million years are good proxy records for understanding past climate. Maximum entropy spectrum of magnectic susceptibility data at the locality of Luochuan, which is in Shaanxi province of China, have shown periodic fluctuations of about $21 \mathrm{kyr}, 41 \mathrm{kyr}, 100 \mathrm{kyr}$ and $400 \mathrm{kyr}$. These periodic fluctuations are related to the long-term variations of earth's orbital elements of the obliquity, the climatic precession and the eccentricity respectively. The data series was separated into two parts to examine the variations of these fluctuations. The first part is from present to $1.1 \mathrm{Myr}$ B.P.( i.e. million years before present ), the second part is from 1.1 Myr B.P. to $2.2 \mathrm{Myr}$ B.P. The periodic fluctuation of $93.4 \mathrm{kyr}$ has the maximum amplitude in the first part of the data series, while the periodic fluctuation of $413 \mathrm{kyr}$ las the maximum amplitude in the second part. The fluctuation about the $400 \mathrm{kyr}$ was found in both parts of the data series.

Cross correlation calculations between the eccentricity data calculated from the paleoclimatic astronomical theory and Luochuan susceptibility data have been performed. The prominent spectral peaks of about 420,123 and $96 \mathrm{kyr}$, which match the main values of the theoritical eccentricity periods, have been found in the cross correlation spectrum. The amount of cross correlation coefficient attained 0.89 after using digital filter with central frequency $410 \mathrm{kyr}$. Susceptibility data has the phase lag about $80 \mathrm{kyr}$.

The fact could be explained by paleoclimatic astronomical theory. Variations of earth's orbital geometry have changed the solar insolation received by Earth in the whole year. Variation of the eccentricity affects the earth's climate by changing the clistance between the Sun and the Earth. The $100 \mathrm{kyr}$ cycle is the mean effect of the eccentricity variations. The dominant peak of $100 \mathrm{kyr}$ in the past $1 \mathrm{Myr}$ may be not only relative to the external forcing, but also to the earth's internal processes.
\end{abstract}

